\title{
NOTE ON CERTAIN ORTHOGONAL POLYNOMIALS ${ }^{1}$
}

\section{DUNHAM JACKSON}

1. Introduction. A well known theorem ${ }^{2}$ states that if $K_{n}(x, t)$ $=\sum_{0}^{n} p_{k}(x) p_{k}(t)$ is the kernel associated with a system of orthonormal polynomials on an interval $(a, b)$ with weight function $\rho(t)$, and if $x_{0}$ is a real number not belonging to the open interval $(a, b)$, the function $K_{n}(x, t)$ is orthogonal to every polynomial of lower degree with respect to $\left|t-x_{0}\right| \rho(t)$ as weight function. This result is to be extended below to orthogonal trigonometric sums, and more generally to other orthogonal polynomials in two real variables on an algebraic curve. ${ }^{3}$ The fact that a single polynomial of the $n$th degree in the original formulation is replaced by two or more sums or polynomials of like degree in the generalized orthogonal systems imparts to the extension some features of novelty. Certain other generalizations are briefly mentioned also.

2. Trigonometric sums. Let $U_{0}(x), U_{1}(x), V_{1}(x), \cdots$ be orthonormal trigonometric sums for weight $\rho(x)$, and let

$$
\begin{aligned}
K_{n}(x, s)=U_{0}(x) U_{0}(s) & +U_{1}(x) U_{1}(s)+\cdots+U_{n}(x) U_{n}(s) \\
& +V_{1}(x) V_{1}(s)+\cdots+V_{n}(x) V_{n}(s) .
\end{aligned}
$$

For definiteness it may be assumed that $U_{k}(x)$ contains no term in $\sin k x$, while $V_{k}(x)$ contains $\sin k x$ with a nonvanishing coefficient; the function $K_{n}(x, s)$ would be unchanged if $U_{k}, V_{k}$ were replaced by any equivalent pair of sums of the $k$ th order. If $T_{n}(x)$ is any trigonometric sum of the $n$th order,

$$
\int_{-\pi}^{\pi} \rho(s) K_{n}(x, s) T_{n}(s) d s=T_{n}(x) .
$$

Suppose $\rho(x) \equiv 0$ throughout an interval $(\alpha, \beta)$. Let $x_{1}, x_{2}$ be any two distinct points of $(\alpha, \beta)$. Let $\tau_{n-1}(x)$ be an arbitrary trigonometric sum of order $n-1$ at most, and let $\sin \frac{1}{2}\left(x-x_{1}\right) \sin \frac{1}{2}\left(x-x_{2}\right) \tau_{n-1}(x)$ $=T_{n}(x)$; the product

$\sin \frac{1}{2}\left(x-x_{1}\right) \sin \frac{1}{2}\left(x-x_{2}\right)=\frac{1}{2} \cos \frac{1}{2}\left(x_{1}-x_{2}\right)-\frac{1}{2} \cos \left[x-\frac{1}{2}\left(x_{1}+x_{2}\right)\right]$

\footnotetext{
1 Presented to the Society, December 29, 1939.

${ }^{2}$ See, e.g., G. Szegö, Orthogonal Polynomials, American Mathematical Society Colloquium Publications, vol. 23, New York, 1939, p. 39.

${ }^{3}$ See D. Jackson, Orthogonal polynomials on plane curves, Duke Mathematical Journal, vol. 3 (1937), pp. 228-236.
} 
is a trigonometric sum of the first order. Then

$$
\begin{aligned}
\int_{-\pi}^{\pi} \rho(s) K_{n}(x, s) \sin \frac{1}{2}(s- & \left.x_{1}\right) \\
= & \sin \frac{1}{2}\left(s-x_{2}\right) \tau_{n-1}(s) d s \\
= & \sin \frac{1}{2}\left(x-x_{1}\right) \sin \frac{1}{2}\left(x-x_{2}\right) \tau_{n-1}(x) .
\end{aligned}
$$

The right-hand member vanishes for $x=x_{1}$ and for $x=x_{2}$; that is to say, $K_{n}\left(x_{1}, s\right)$ and $K_{n}\left(x_{2}, s\right)$ as trigonometric sums of the nth order in $s$ are orthogonal to every sum $\tau_{n-1}(s)$ of lower order, with respect to $\sin \frac{1}{2}\left(s-x_{1}\right) \sin \frac{1}{2}\left(s-x_{2}\right) \rho(s)$ as weight function. This weight function is of constant sign, since $\rho(s)$ vanishes throughout the interval $\left(x_{1}, x_{2}\right)$.

The sums $K_{n}\left(x_{1}, s\right)$ and $K_{n}\left(x_{2}, s\right)$ are linearly independent. An identity $K_{n}\left(x_{2}, s\right)=C K_{n}\left(x_{1}, s\right)$ would require in particular that $U_{0}\left(x_{2}\right)$ $=C U_{0}\left(x_{1}\right), \quad U_{1}\left(x_{2}\right)=C U_{1}\left(x_{1}\right), \quad V_{1}\left(x_{2}\right)=C V_{1}\left(x_{1}\right)$, that is, that $C=1$, $\cos x_{2}=\cos x_{1}, \sin x_{2}=\sin x_{1}$.

In the limiting case $x_{2}=x_{1}$,

$$
\int_{-\pi}^{\pi} \rho(s) K_{n}(x, s) \sin ^{2} \frac{1}{2}\left(s-x_{1}\right) \tau_{n-1}(s) d s=\sin ^{2} \frac{1}{2}\left(x-x_{1}\right) \tau_{n-1}(x)
$$

and by differentiation with respect to $x$ under the sign of integration

$$
\begin{aligned}
& \int_{-\pi}^{\pi} \rho(s) K_{n x}(x, s) \sin ^{2} \frac{1}{2}\left(s-x_{1}\right) \tau_{n-1}(s) d s \\
& \quad=\sin \frac{1}{2}\left(x-x_{1}\right) \cos \frac{1}{2}\left(x-x_{1}\right) \tau_{n-1}(x)+\sin ^{2} \frac{1}{2}\left(x-x_{1}\right) \tau_{n-1}^{\prime}(x),
\end{aligned}
$$

where $K_{n x}(x, s)$ means $(\partial / \partial x) K_{n}(x, s)$. Both right-hand members vanish for $x=x_{1} ; K_{n}\left(x_{1}, s\right)$ and $K_{n x}\left(x_{1}, s\right)$ as trigonometric sums of the $n$th order in $s$ are orthogonal to every sum of lower order for weight $\sin ^{2} \frac{1}{2}\left(s-x_{1}\right) \rho(s)$. Here no assumption with regard to vanishing of $\rho$ is required. It is obvious that $K_{n}$ and $K_{n x}$ are linearly independent, for one contains a constant term and the other does not.

Vanishing of $\rho$ is again unnecessary if $x_{1}$ and $x_{2}$ are allowed to be complex. Let $x_{1}=\gamma+i \delta, x_{2}=\gamma-i \delta$, with $\gamma$ and $\delta$ real,

$$
\begin{aligned}
\sin \frac{1}{2}\left(x-x_{1}\right) \sin \frac{1}{2}\left(x-x_{2}\right) & =\frac{1}{2} \cos i \delta-\frac{1}{2} \cos (x-\gamma) \\
& =\frac{1}{2} \cosh \delta-\frac{1}{2} \cos (x-\gamma) .
\end{aligned}
$$

Since each member of (1) represents a trigonometric sum in $x$, and they are identical for real values of $x$, they are equal also when $x$ is complex. For $x=x_{1}$ the right-hand member is zero, and the real and pure imaginary parts of the expression on the left must vanish separately. The same result is obtained by setting $x=x_{2}$. The real and pure imaginary parts of $K_{n}\left(x_{1}, s\right)$ for real $s$ (or of its conjugate $K_{n}\left(x_{2}, s\right)$ ) are 
orthogonal to every trigonometric sum of lower order in $s$ with respect to the non-negative weight function $\left[\frac{1}{2} \cosh \delta-\frac{1}{2} \cos (s-\gamma)\right] \rho(s)$. These real and pure imaginary components, being linearly independent combinations of the linearly independent functions $K_{n}\left(x_{1}, s\right)$ and $K_{n}\left(x_{2}, s\right)$, are themselves linearly independent trigonometric sums of the $n$th order in $s$.

3. Polynomials on an algebraic curve. Orthogonal trigonometric sums can be regarded as a special instance of orthogonal polynomials in two real variables on an algebraic curve, the curve in this case being a circle (or, without essentially greater generality, an ellipse). Considerations analogous to those outlined above apply to the more general problem. As an illustration the reasoning will be formulated for a curve of the third degree. It will be immediately apparent that the same type of argument can be applied to curves of higher degree, if exceptional cases are avoided, or, on the other hand, to an alternative treatment of orthogonal polynomials on a circle, or to orthogonal polynomials on hyperbolic arcs. (Orthogonal polynomials on a parabola are essentially orthogonal polynomials in a single variable.)

Let $C$ be a curve of the third degree. Let $\rho(x, y)$ be a non-negative weight function defined on $C$, and, for simplicity, vanishing identically except on an arc or arcs of finite extent. Let $p_{01}(x, y), p_{11}(x, y)$, $p_{12}(x, y)$, and, for $n \geqq 2, p_{n 1}(x, y), p_{n 2}(x, y), p_{n 3}(x, y)$ denote the members of a corresponding system of orthonormal polynomials on $C$, each polynomial being of total degree indicated by its first subscript. Let

$$
K_{n}(x, y, u, v)=\sum_{k \leqq n} p_{k j}(x, y) p_{k j}(u, v),
$$

the summation being extended over all the relevant pairs of subscripts. As in the special case of trigonometric sums, the function $K_{n}$ is completely determinate when the points $(x, y)$ and $(u, v)$ are on the curve $C$ in their respective planes, although the individual polynomials of any specified degree in the system admit orthogonal transformation among themselves.

Let $a x+b y+c=0$ be the equation of a line intersecting $C$ in three distinct points $\left(x_{1}, y_{1}\right),\left(x_{2}, y_{2}\right),\left(x_{3}, y_{3}\right)$, and so situated that the domain of orthogonality of the polynomials is all on one side of it, that is, so that $a x+b y+c$ is of constant sign where $\rho \neq 0$. The intersections may be real, or two of them may be conjugate complex; it is understood that the coefficients $a, b, c$, as well as those in the equation of $C$, are real. It is to be understood further, if $C$ is degenerate, that the domain of orthogonality includes a portion of positive measure 
(with respect to arc length as variable of integration) on each of the loci defined by the vanishing of an irreducible factor in the equation of $C$.

If $P_{n}(x, y)$ is any polynomial of the $n$th or lower degree, then for $(x, y)$ on $C$

$$
\int_{C} \rho(u, v) K_{n}(x, y, u, v) P_{n}(u, v) d s=P_{n}(x, y),
$$

where $s$ denotes arc length on the curve $C$ in the $(u, v)$ plane. While the identity holds in general only on the curve, it is valid at all points of the curve, not merely where $\rho \neq 0$. If $\pi_{n-1}(x, y)$ is an arbitrary polynomial of degree $n-1$ at most, and $P_{n}(x, y)=(a x+b y+c) \pi_{n-1}(x, y)$, the identity states that

$$
\begin{aligned}
\int_{C} \rho(u, v) K_{n}(x, y, u, v)(a u+b v+c) & \pi_{n-1}(u, v) d s \\
& =(a x+b y+c) \pi_{n-1}(x, y) .
\end{aligned}
$$

Since the points $\left(x_{\nu}, y_{\nu}\right), \nu=1,2,3$, lie on the curve and at the same time on the line $a x+b y+c=0$, the relation holds with the right-hand member equal to zero if any one of these pairs of values is substituted for $(x, y)$. The functions $K_{n}\left(x_{\nu}, y_{\nu}, u, v\right)$ as polynomials in $u$ and $v$ are orthogonal to every polynomial of lower degree for weight $(a u+b v+c) \rho(u, v)$.

The three polynomials $K_{n}\left(x_{\nu}, y_{\nu}, u, v\right)$ are linearly independent on $C$ if $n \geqq 2$. For, as the polynomials $p_{k j}(u, v)$ are linearly independent on the curve, a relation of linear dependence of the form $\sum_{\nu} C_{\nu} K_{n}\left(x_{\nu}, y_{\nu}, u, v\right)=0$ would imply corresponding relations of linear dependence among the quantities $p_{k j}\left(x_{\nu}, y_{\nu}\right)$ as coefficients in the $K$ 's. In particular, the first six of the equations $\sum_{\nu} C_{\nu} p_{k j}\left(x_{\nu}, y_{\nu}\right)$ would require that

$$
\begin{aligned}
C_{1}+C_{2}+C_{3} & =0, \\
C_{1} x_{1}+C_{2} x_{2}+C_{3} x_{3} & =0, \\
C_{1} y_{1}+C_{2} y_{2}+C_{3} y_{3} & =0, \\
C_{1} x_{1}^{2}+C_{2} x_{2}^{2}+C_{3} x_{3}^{2} & =0, \\
C_{1} x_{1} y_{1}+C_{2} x_{2} y_{2}+C_{3} x_{3} y_{3} & =0, \\
C_{1} y_{1}^{2}+C_{2} y_{2}^{2}+C_{3} y_{3}^{2} & =0 .
\end{aligned}
$$

The first, second, and fourth of these equations are possible with $C$ 's not all zero only if two of the $x$ 's are equal, and the first, third, and 
sixth only if two of the $y$ 's are equal. But this is inconsistent with the hypothesis that $\left(x_{1}, y_{1}\right),\left(x_{2}, y_{2}\right),\left(x_{3}, y_{3}\right)$ are three distinct points on a straight line. Similarly, though more simply, any two of the polynomials $K_{1}\left(x_{\nu}, y_{\nu}, u, v\right)$ are linearly independent on $C$. The expressions $K_{n}\left(x_{\nu}, y_{\nu}, u, v\right)$ give the complete system of orthogonal polynomials corresponding to the weight function $(a x+b y+c) \rho(x, y)$. If two of the points $\left(x_{\nu}, y_{\nu}\right)$ are conjugate complex, real polynomials for the orthogonal system can be obtained by taking the real and pure imaginary parts of the corresponding $K$ 's.

Similar reasoning is clearly effective for a curve of the $N$ th degree with a straight line meeting it in $N$ distinct points. For a treatment of certain cases of coincident points of intersection the discussion will be limited to the curve of the third degree.

Suppose now that the line $a x+b y+c=0$, instead of meeting the curve in three distinct points, is tangent to it at an ordinary point $\left(x_{1}, y_{1}\right)$, and intersects it at a point $\left(x_{2}, y_{2}\right)$ distinct from $\left(x_{1}, y_{1}\right)$. It is still assumed that the part of the curve on which $\rho \neq 0$ is all on one side of the line, that is, that $a x+b y+c$ is of constant sign there. At least one of the coefficients $a, b$ is different from zero; without loss of generality it may be supposed that $b \neq 0$, that is, that the line is not parallel to the $y$-axis. In the neighborhood of the point $\left(x_{1}, y_{1}\right)$, $y$ is a single-valued function of $x$ on the curve, with a derivative whose value reduces at $\left(x_{1}, y_{1}\right)$ to $(-a / b)$, the slope of the tangent line. In this neighborhood on $C$, by differentiation of (2),

$$
\begin{aligned}
\int_{C} \rho(u, v) K_{n x}(x, y, & u, v)(a u+b v+c) \pi_{n-1}(u, v) d s \\
& =\frac{d}{d x}\left[(a x+b y+c) \pi_{n-1}(x, y)\right] \\
& =(a x+b y+c) \frac{d}{d x} \pi_{n-1}(x, y)+\left(a+b y^{\prime}\right) \pi_{n-1}(x, y),
\end{aligned}
$$

where $K_{n x}(x, y, u, v)$ means the total derivative of $K_{n}$ with respect to $x$, equal to $\partial K_{n} / \partial x+y^{\prime} \partial K_{n} / \partial y$. At $\left(x_{1}, y_{1}\right)$ both $a x+b y+c$ and $a+b y^{\prime}$ vanish. The function $K_{n x}\left(x_{1}, y_{1}, u, v\right)$, which is still a polynomial as regards its dependence on $u$ and $v$, is orthogonal to $\pi_{n-1}(u, v)$ for weight $(a u+b v+c) \rho(u, v)$.

There have been obtained then for $n \geqq 2$ three polynomials $K_{n}\left(x_{1}, y_{1}, u, v\right), K_{n x}\left(x_{1}, y_{1}, u, v\right), K_{n}\left(x_{2}, y_{2}, u\right.$, v), each possessing the property of orthogonality. These are once more linearly independent on the curve. Without effect on the form of $K_{n}$, and so without any impairment of generality, it may be assumed that the first six poly- 
nomials in the orthogonal system are specifically those obtained by applying the Schmidt process to the monomials $1, x, y, x^{2}, x y, y^{2}$, taken in that order. Then $p_{11}(x, y)$ does not involve $y$, and $p_{21}(x, y)$ involves neither $x y$ nor $y^{2}$. Suppose there were an identity of the form

$$
C_{1} K_{n}\left(x_{1}, y_{1}, u, v\right)+C_{2} K_{n x}\left(x_{1}, y_{1}, u, v\right)+C_{3} K_{n}\left(x_{2}, y_{2}, u, v\right)=0
$$

with $C$ 's not all zero. Then

$$
C_{1} p_{k j}\left(x_{1}, y_{1}\right)+C_{2} p_{k j}^{\prime}\left(x_{1}, y_{1}\right)+C_{3} p_{k j}\left(x_{2}, y_{2}\right)=0
$$

for each pair of indices $(k, j)$ in which $k \leqq n$, the accent indicating total differentiation with respect to $x$. The equations for the first four pairs of indices may be simplified to the form

$$
\begin{aligned}
C_{1}+C_{3} & =0, \\
C_{1} x_{1}+C_{2}+C_{3} x_{2} & =0 \\
C_{1} y_{1}-C_{2} a / b+C_{3} y_{2} & =0 \\
C_{1} x_{1}^{2}+2 C_{2} x_{1}+C_{3} x_{2} & =0 .
\end{aligned}
$$

The first, second, and fourth of these equations require that $x_{1}=x_{2}$, which is impossible under the hypothesis that $\left(x_{1}, y_{1}\right)$ and $\left(x_{2}, y_{2}\right)$ are distinct and $b \neq 0$. It is immediately apparent that any two of the three $K$ 's are linearly independent for $n=1$. So the $K$ 's give the complete orthogonal system for weight $(a x+b y+c) \rho(x, y)$.

Finally, let the line meet the curve at a double point $\left(x_{1}, y_{1}\right)$ at which there are distinct tangents with finite slopes, and at another point $\left(x_{2}, y_{2}\right)$. It is assumed, as always, that $a x+b y+c$ is of constant sign where $\rho$ does not vanish. Let $K_{1}(u, v)$ and $K_{2}(u, v)$ be the total derivatives $K_{n x}\left(x_{1}, y_{1}, u, v\right)$ calculated respectively for the two branches of the curve through $\left(x_{1}, y_{1}\right)$; let the corresponding slopes be $\lambda_{1}$ and $\lambda_{2}$. Then

$$
\begin{aligned}
\int_{C} \rho(u, v) K_{\nu}(u, v)(a u+b v+c) & \pi_{n-1}(u, v) d s \\
& =\left(a+b \lambda_{\nu}\right) \pi_{n-1}\left(x_{1}, y_{1}\right), \quad \nu=1,2 .
\end{aligned}
$$

It is seen that $\left(a+b \lambda_{2}\right) K_{1}(u, v)-\left(a+b \lambda_{1}\right) K_{2}(u, v)$ is orthogonal to $\pi_{n-1}(u, v)$ for the composite weight function. This polynomial and the two polynomials $K_{n}\left(x_{1}, y_{1}, u, v\right), K_{n}\left(x_{2}, y_{2}, u, v\right)$ are found to be linearly independent on $C$ for $n \geqq 2$, and once more a complete orthogonal system is obtained.

These illustrations will be allowed to suffice for the case of multiple intersections. 
4. Other cases. The corresponding theory for a non-algebraic curve is complicated by the fact that the number of polynomials of the $n$th degree in the orthogonal system increases with $n$, but is compensatingly simplified by the observation that the representation corresponding to (2) holds for all values of $x$ and $y$, so that (except for the assumption that the domain of orthogonality is all on one side of the line) the points where the straight line meets the curve are no longer a matter of special concern. If $\left(x_{\nu}, y_{\nu}\right)$ are any $n+1$ distinct points on the line, independent polynomials of the $n$th degree orthogonal to every polynomial of lower degree with respect to the composite weight function are given by $K_{n}\left(x_{\nu}, y_{\nu}, u, v\right)$.

A similar conclusion holds for orthogonality on a two-dimensional region.

The University of Minnesota

\section{NOTE ON AN INEQUALITY OF STEINER ${ }^{1}$}

\section{T. RADÓ AND P. REICHELDERFER}

Let $Q$ denote the unit square $0 \leqq x, y \leqq 1$. If $f(x, y)$ be any function defined and continuous on $Q$, the relation $z=f(x, y)$ yields a continuous surface defined over $Q$. The Lebesgue area ${ }^{2}$ of this surface will be denoted by $L(f)$. Let $z=f_{1}(x, y), z=f_{2}(x, y)$ be two continuous surfaces defined over $Q$; then clearly $z=\left[f_{1}(x, y)+f_{2}(x, y)\right] / 2$ is a continuous surface defined over $Q$. The inequality of Steiner ${ }^{3}$ states that $L\left(\left[f_{1}+f_{2}\right] / 2\right) \leqq\left[L\left(f_{1}\right)+L\left(f_{2}\right)\right] / 2$. McShane ${ }^{4}$ obtained interesting and important results concerning the situation where the sign of equality holds in this relation. In this note we improve his results and, in a sense, give them a final form.

In order to emphasize and to clarify what is significant and interesting in the results of McShane and in our improvements thereon, we remind our reader of a few facts concerning the Lebesgue area. ${ }^{5}$ Given a continuous surface $z=f(x, y)$ defined over $Q$; if $L(f)$ is finite then the partial derivatives $f_{x}$ and $f_{y}$ exist almost everywhere in $Q$, the integral $\iint_{Q}\left[1+f_{x}^{2}+f_{y}^{2}\right]^{1 / 2} d x d y$ exists, and the relation

1 Presented to the Society, April 13, 1940.

${ }^{2}$ See S. Saks, Theory of the Integral, Warsaw and Lwów, 1937, chap. 5, for the facts used in this paper concerning the Lebesgue area.

${ }^{3}$ E. J. McShane, On a certain inequality of Steiner, Annals of Mathematics, (2), vol. 33 (1932), pp. 125-138.

${ }^{4}$ Loc. cit. ${ }^{3}$

${ }^{5} \mathrm{Cf}^{2}$ 